\title{
Effects of tea polyphenols and EGCG on glucose metabolism and intestinal flora in diabetic mice fed a cornstarch-based functional diet
}

\author{
Jun LIU ${ }^{1}$ (D), Yang-Jun LV ${ }^{1}$, Jun-Xian PAN ${ }^{1}$, Yu-Lan JIANG ${ }^{1}$, Yue-Jin ZHU ${ }^{1}$, Shi-Kang ZHANG ${ }^{1 *}$
}

\begin{abstract}
Diabetes is a worldwide metabolic diseases that requires adjustments in the blood glucose. Cornstarch is one kind of resistant starch, which helps to control postprandial glucose, and is a desired alternatives of carbohydrates for developing functional food. Herein, a cornstarch-based functional diet (CFD) was developed, and in this study we investigated the effects of CFD supplemented with tea polyphenols and EGCG on blood glucose and intestinal flora in diabetic mice. The results showed that diabetic mice had significant weight loss and serious hyperglycemia. The CFD or CFD supplemented with tea polyphenols and EGCG resulted in a trend toward reduced fasting blood glucose. Furthermore, diabetes had significant intestinal flora disturbance, with particularly increased levels of Bacteroidaceae, Helicobacteraceae, Ruminococcaceae, Enterobacteriaceae, Rikenellaceae and Saccharibacteria_genera_incertae_sedis, and decreased Prevotellaceae, Coriobacteriaceae, Bifidobacteriaceae and Verrucomicrobiaceae. Interesting, CFD supplemented with EGCG significantly increased levels of Prevotellaceae and Coriobacteriaceae, and decreased Lachnospiraceae, Helicobacteraceae, Ruminococcaceae and Saccharibacteria_genera_incertae_sedis compared to controls. CFD supplemented with tea polyphenols significantly increased the Coriobacteriaceae and Bifidobacteriaceae. The comprehensive analysis indicated that tea polyphenols and EGCG supplementation improved glucose metabolism, and reversed the sick changes of intestinal flora, suggesting they are two valuable additions to promote nutritional value of CFD.
\end{abstract}

Keywords: diabetes; functional food; intestinal flora; tea polyphenols; $16 \mathrm{~S}$ rDNA sequencing.

Pratical Application: Regulate intestinal flora balance in patients with type 2 diabetes mellitus using tea functional diet.

\section{Introduction}

Diabetes is a worldwide disease. Poorly controlled frequent high blood glucose leads to the long-term complications of diabetes, including oxidative stress (Damasceno et al., 2002), glucolipid metabolic disorder (Stefano et al., 2016). It is well-known that ordinary starchy food is a threat to diabetes patients, but resistant starch may be a desired alternatives (Nugent, 2005). Resistant starch is a new resource of dietary fiber, and is the portion of ingested starchy material which escapes digestion in the small intestine. Cornstarch is one kind of resistant starch, and it contains more than $50 \%$ content of resistant starch (Nugent, 2005; Zhang et al., 2018a). Therefore, we can use cornstarch to develop a new kind of functional food for controlling postprandial glucose. Most importantly, our previous studies showed that tea products (tea polyphenols, epigallocatechin gallate (EGCG, one major component of tea polyphenols), matcha or instant tea) exerted great inhibitory effects on the hydrolysis of cornstarch in vitro (Zhang et al., 2018a), suggesting tea products may be a desired food supplement for developing cornstarch-based functional food.

Intestinal flora is closely related to health and metabolism, and intestinal micro-ecological disorder cause a series of physical dysfunction. Many studies showed that diabetes had serious intestinal flora disturbance (Zhang et al., 2020; Lippert et al., 2017;
Gomes et al., 2018; Baothman et al., 2016), thus regulating the intestinal flora balance may serve as a pathway to improvement in diabetes. Polyphenolic compounds are the main functional active ingredients in tea, including tea polyphenols and its monomer EGCG. They have many health benefits, such as inhibiting the activity of amylase and glucosidase (Qiu et al., 2017), reducing insulin resistance (Lin \& Lin, 2008), inhibiting lipid absorption (Nakai et al., 2005) enhancing fat oxidation (Sae-Tan et al., 2011), and inhibiting gluconeogenesis (Collins et al., 2007). Therefore, a cornstarch-based functional diet (CFD) was developed, and in this study we deeply investigated and studied the effects of such a diet supplemented with tea polyphenols and EGCG on blood glucose and intestinal flora in diabetic mice, aimed to providing basic theoretical information for developing new functional food.

\section{Materials and methods}

\subsection{Materials}

One-month-old Female Kunming mice (body weight $20 \pm 3$ g) was provided by the Animal Experiment Center, Zhejiang Academy of Medical Sciences (Hangzhou, China). Basal feed was purchased from Jiangsu Collaborative Bioengineering co. 
LTD (Nanjing, China), which containing $18.6 \%$ crude protein, $4.8 \%$ crude fat, $61 \%$ carbohydrate.

Cornstarch was purchased from Qinhuangdao Lihua Starch Co., Ltd (Qinghuangdao, China). Potassium dihydrogen phosphate and calcium carbonate were purchased from Lianyungang Youjin Food Additive Technology Development Co., Ltd (Lianyungang, China). Other dietary materials, like butter, cake flour and meringue was purchased from local supermarket.

EGCG (95\% purify) and tea polyphenols (total polyphenols contents of $90 \%$ ) were purchased from Ebeikar Tea \& Extracts Co., Ltd. (Hangzhou, China).

\subsection{Effects of tea polyphenols and EGCG on cornstarch metabolism in vivo}

Normal mice were randomly allotted into three treatments $(\mathrm{n}=10)$ after subjected to 4 -week basal diet. Control group C, test groups A and B were intragastrically administered (2.2 g/ $\mathrm{kg}$ body weight) with cornstarch, cornstarch supplemented with $0.5 \%$ tea polyphenols or with $0.5 \%$ EGCG, respectively. Levels of postprandial blood glucose was detected at $0,15,30$, 60, 90 and 120 min with a quick blood sugar radiometer after gavage administration (Roche Diagnostics Products (Shanghai) Co. LTD.).

\subsection{Effects of tea polyphenols and EGCG on blood glucose and intestinal flora in diabetic mice}

\section{Experimental design, and feeding management}

A mouse model with diabetes mellitus was established by alloxan ( $45 \mathrm{mg} / \mathrm{kg}$ body weight), and then was randomly allotted into four treatments ( $n=8$ or 10 ), group D-C fed a CFD, D-B fed a basal diet, test groups D-T, D-E fed a CFD supplemented $0.48 \%$ tea polyphenols or EGCG, respectively. Normal mice was treated with stroke-physiological saline solution, and then was recognized as control group N-C $(n=10)$, fed a CFD.

The CFD consists of $20.69 \%$ cornstarch, $25.33 \%$ butter, $20.69 \%$ cake flour, $16.68 \%$ meringue powder, $8.66 \%$ dextrin, $4.86 \%$ egg yolk, $1.82 \%$ potassium dihydrogen phosphate and $1.27 \%$ calcium carbonate. And it contained $22.1 \%$ fat, $20.93 \%$ protein and $40.94 \%$ carbohydrate, and had an energy content of $19.18 \mathrm{~kJ} / \mathrm{g}$.

All the mice were raised under the same conditions, and housed in a temperature-controlled room at $24{ }^{\circ} \mathrm{C} \pm 1{ }^{\circ} \mathrm{C}$ and $50 \% \pm 5 \%$ relative humidity on a 12 -h light/dark cycle. This study was approved by the ethics committee of Zhejiang University (Hangzhou, China), amd all the experimental procedures were approved by Zhejiang University Institutional Animal Care and Use Committee.

Intestinal flora play a significant role in health and disease, and faeces reflect the ultimate result of interaction of entire intestinal flora and host. When simultaneously subjected to a 4-week CFD, the fecal samples from N-C, D-C, D-E and D-T were collected, and the changes of intestinal flora were analyzed by $16 \mathrm{~S}$ rDNA sequencing (Zhang et al., 2018b). Fasting blood glucose (FBG) were detected with a quick blood sugar radiometer after gavage (Roche Diagnostics Products (Shanghai) Co. LTD.).

\subsection{DNA extraction, $16 \mathrm{~S}$ rDNA gene sequencing and data quality control and data analysis}

DNA was extracted using an E.Z.N.A. Stool DNA Kit (D4015-02, Omega Inc., USA) according to the manufacturer's instructions. The $\mathrm{V} 4$ region of the gene encoding the prokaryotic small (16S) rRNA subunit was amplified with slightly modified versions of primers 515f (5'-GTGYCAGCMGCCGCGGTAA-3') and 806R (5'-GGACTACNVGGGTWTCTAAT-3') (Huang et al., 2013). The 5' ends of the primers were tagged with specific barcodes and universal sequencing primers. PCR amplification was performed with $25 \mu \mathrm{L}$ reaction-mixture aliquots containing 50 ng of template DNA, $12.5 \mu \mathrm{L}$ pusion host start flex $2 \mathrm{X}$ master mix (M0536L, Shanghai Yitao Biological Instrument co., LTD, China), $2.5 \mu \mathrm{L}$ of forward primer, $2.5 \mu \mathrm{L}$ of reverse primer, and PCR-grade water to adjust the volume. PCR amplification procedure were carried out with PCR Amplifier (A200, Hangzhou Langji Scientific Instrument Co. LTD, China), and the specific PCR conditions were as follows: an initial denaturation at $98^{\circ} \mathrm{C}$ for $30 \mathrm{~s}$, followed by 35 cycles of denaturation at $98^{\circ} \mathrm{C}$ for $10 \mathrm{~s}$, annealing at $54^{\circ} \mathrm{C}$ for $30 \mathrm{~s}$, and extension at $72{ }^{\circ} \mathrm{C}$ for $45 \mathrm{~s}$, and a final extension at $72{ }^{\circ} \mathrm{C}$ for $10 \mathrm{~min}$. PCR products were confirmed by $2 \%$ agarose gel electrophoresis, purified using AMPure XT breads (Beckman Coulter Genomics, Danvers, MA, USA), and quantified by Qubit (Invitrogen, USA).

Amplicon pools were prepared for sequencing, and library size and quality were assessed using an Agilent 2100 Bioanalyzer (Agilent, USA) and a Library Quantification Kit for Illumina (Kapa Biosciences, Woburn, MA, USA), respectively. The PhiX control library v3 (Illumina) and the amplicon library were combined except for a $30 \%$ PhiX spike-in. The libraries were sequenced using $2500 \mathrm{MiSeq}$ runs. Additionally, one library was sequenced by both this method and using standard Illumina sequencing primers, eliminating the need for a third (or fourth) index read.

Samples were sequenced on an Illumina MiSeq platform according to the manufacturer's recommendations. Pairedend reads was assigned to samples based on their unique barcode and truncated by removing the barcode and primer sequence. Paired-end reads were merged using FLASH software (https://ccb.jhu.edu/software/FLASH/). Quality filtering of raw tags was performed under specific filtering conditions to obtain high-quality clean tags according to FastQC (V0.10.0) (Babraham Bioinformatics, https://www.bioinformatics.babraham. ac.uk/projects/fastqc/). Chimeric sequences were filtered using Vsearch software (v2.3.4) (https://github.com/torognes/vsearch) (Rognes et al., 2016). Sequences with $\geq 97 \%$ similarity were assigned to the same operational taxonomic units (OTUs) using Vsearch (v2.3.4). Representative sequences were chosen for each OTU, and taxonomic data were then assigned to each representative sequence using Ribosomal Database Project (RDP) classifier (https://rdp.cme.msu.edu/classifier/classifier.jsp;jsess ionid=6EAF5285A39E3DC70FAEB431CBCEC920.radiant). Determination of differences between dominant species in different groups and multiple sequence alignment were conducted using PyNAST software (http://biocore.github.io/pynast/) to study 
phylogenetic relationships of different OTUs (Caporaso et al. 2010). The levels of OTUs was normalised relative to the sample with the fewest sequences.

\subsection{Statistical analysis}

Figures were made by GraphPad Prism (version 6), and the differences between treatments were analyzed by using Nonparametric Kruskal-wallis's test in SPSS (version 24.0). $p$-values $<0.05$ were considered significant.

\section{Results and discussion}

\subsection{Effects of tea polyphenols and EGCG on cornstarch metabolism in vivo}

As Figure 1 shows, the concentrations of postprandial blood glucose in group C reached the highest level of $9.19 \mathrm{mmol} / \mathrm{L}$ at 30 minutes. However, the concentrations of postprandial blood glucose in group A and B reached the highest level of $6.58 \mathrm{mmol} / \mathrm{L}$ at 30 minutes, $5.42 \mathrm{mmol} / \mathrm{L}$ at 60 minutes, respectively. The results suggested that tea polyphenols and EGCG exerted greater inhibitory effects on the digestion and absorption of cornstarch in vivo. Tea polyphenols and EGCG not only influenced and reduced the big blood glucose spikes, but also delayed the time to reach the levels of peak.

As we know, cornstarch is a kind of resistant starch (Zhang et al., 2018a), but eating cornstarch still cause big changes of postprandial blood glucose. Interestingly enough, tea polyphenols and EGCG exerted great inhibitory effects on the digestion and absorption of cornstarch in vivo. And this findings were consistent with our previous conclusions. Our previous test in vitro illustrated that $0.5 \%$ EGCG and $0.5 \%$ tea polyphenols supplementation were capable of reducing the hydrolysis rate of cornstarch by $25.1 \%$ and $20.6 \%$, respectively. And this phenomenon were related to the inhibitory effects on the amylase activity (Zhang et al., 2018a). Therefore, the results confirmed that tea polyphenols and EGCG have great a potentiality to be used in developing cornstarch-based functional food.

\subsection{Effects of tea polyphenols and EGCG on blood glucose and intestinal flora in diabetic mice}

\section{Analysis of feed intake and body weight changes}

As Figure 2 shows, all the diabetic mice had significant weight loss and hyperphagia, particularly in group D-C. These findings were consistent with Namkoong et al. (2005) study. Significantly, the body weight levels in group D-C ( $23-28 \mathrm{~g} / \mathrm{mice})$ were the lowest, but they consumed the highest feed intake level $(8-15 \mathrm{~g} / \mathrm{mice} / \mathrm{d})$. From the highest to lowest performance, the body weights in this study were ranked in the following order, $\mathrm{N}-\mathrm{C}>\mathrm{D}-\mathrm{E}, \mathrm{D}-\mathrm{T}, \mathrm{D}-\mathrm{B}>\mathrm{D}-\mathrm{C}$. The feed intakes were: $\mathrm{D}-\mathrm{C}>\mathrm{D}-\mathrm{E}$, $\mathrm{D}-\mathrm{T}, \mathrm{D}-\mathrm{B}>\mathrm{N}-\mathrm{C}$. The body weight and feed intake levels between test groups $\mathrm{D}-\mathrm{T}$ and $\mathrm{D}$-E were not clearly different.

\section{Analysis of FBG changes}

As Figure 3 shows, the concentrations of FBG in group D-B were the highest $(28-32 \mathrm{mmol} / \mathrm{L})$, while in group $\mathrm{N}-\mathrm{C}$ were the lowest (3-4 mmol/L). The FBG levels in diabetic groups D-C, D-T and D-E were obviously lower than that of diabetic group D-B.

The results of this study showed that CFD supplemented with tea polyphenols and EGCG resulted in a trend toward increased body weight levels, but reduced feed intake level. Moreover, CFD alone or supplemented with tea polyphenols and EGCG exerted a trend towards decreased FBG. The likely explanation may be related to cornstarch, tea' functional ingredients, or both participate. As we know, cornstarch contains a high levels of resistant starch (> 50\%), and has lower kinetic constants and slower digestion than other types of starch, which helps to inhibit the release of glucose (Nugent, 2005; Zhang et al., 2018a). Besides, tea's functional ingredients may inhibit starch digestion and absorption. In this study, the purity of the EGCG used in

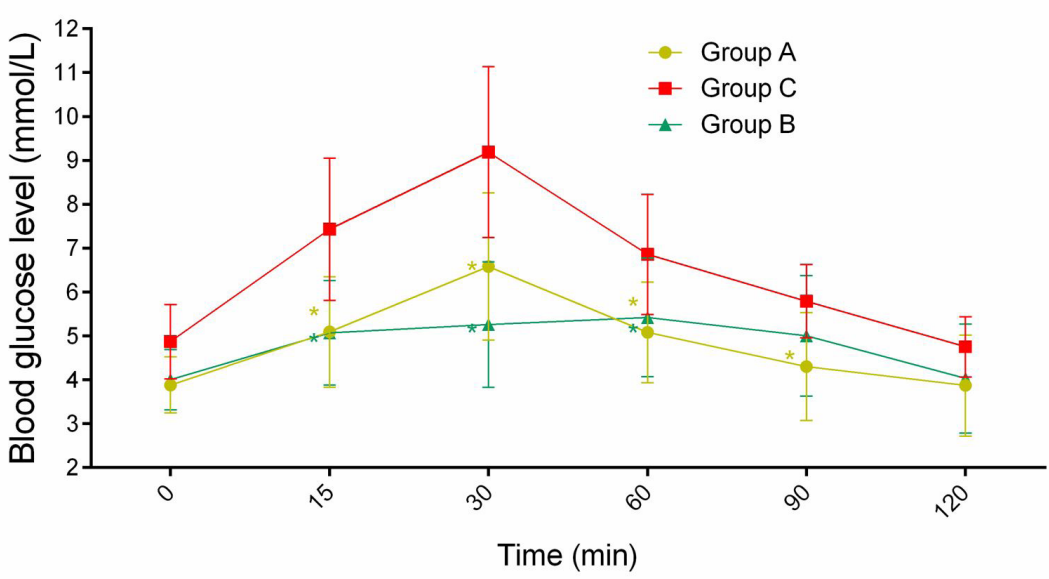

Figure 1. Effects of tea products on digestion and absorption of cornstarch in normal mice. Note: yellow colored "**" and green colored "*»" respectively represent significant difference were found in cornstarch with $0.5 \%$ tea polyphenols, cornstarch with $0.5 \%$ EGCG treatments when compared with cornstarch administration by gavage $(p<0.05)$. Control group C, test groups A and B were intragastrically administered $(2.2 \mathrm{~g} /$ $\mathrm{kg}$ body weight) with cornstarch, cornstarch supplemented with $0.5 \%$ tea polyphenols, cornstarch supplemented with $0.5 \%$ EGCG, respectively. 

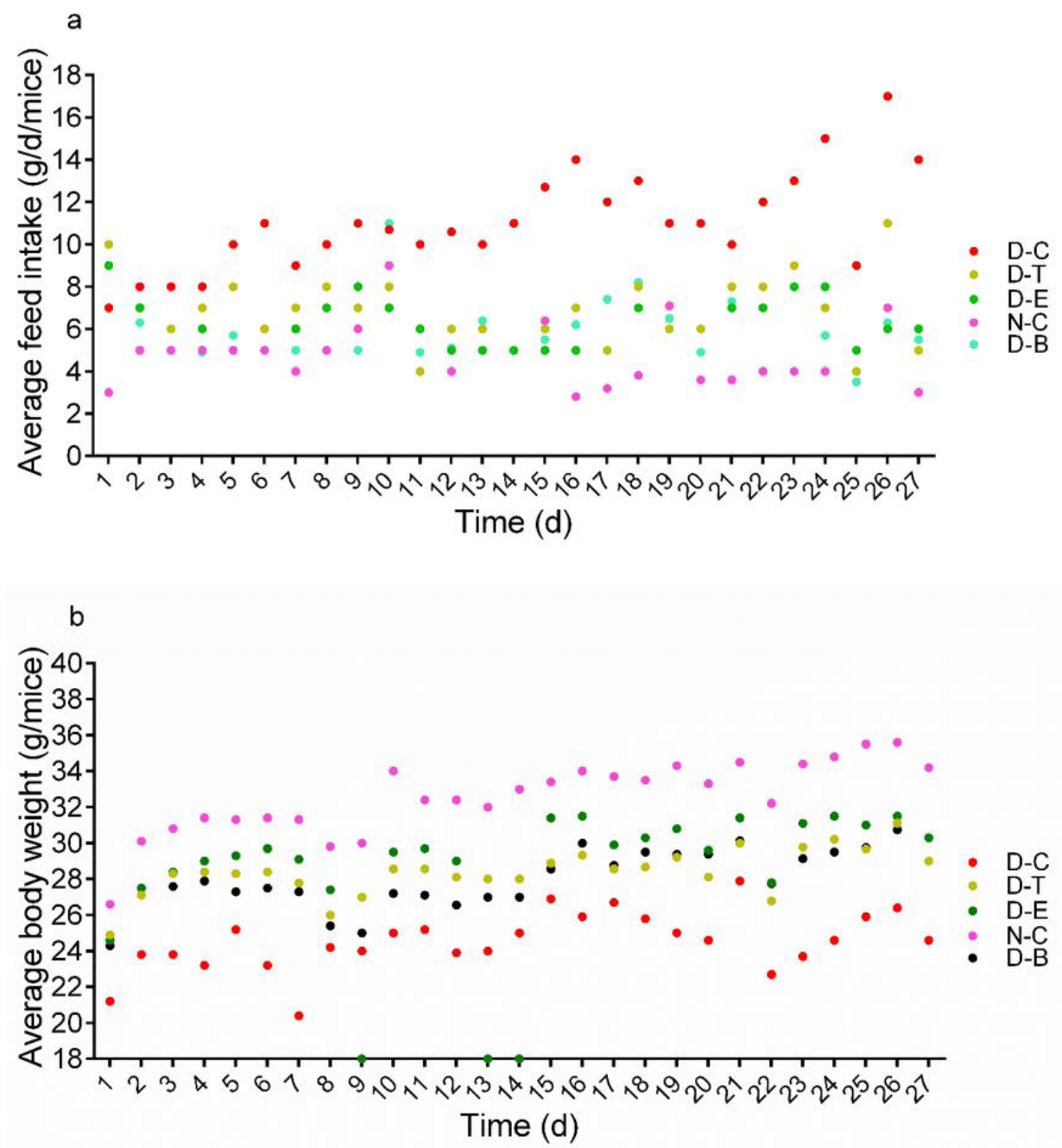

Figure 2. Effects of experimental diet supplemented with tea products on feed intake and body weight of diabetic mice. Note: Mice in diabetic group D-C fed a cornstarch-based diet, D-T fed a cornstarch-based functional diet supplemented with tea polyphenols, group D-E fed a cornstarch-based functional diet supplemented with EGCG, group D-B fed a basal diet, respectively $(\mathrm{n}=8$ or 10$)$. Mice in normal group N-C fed a cornstarch-based functional diet $(\mathrm{n}=10)$.

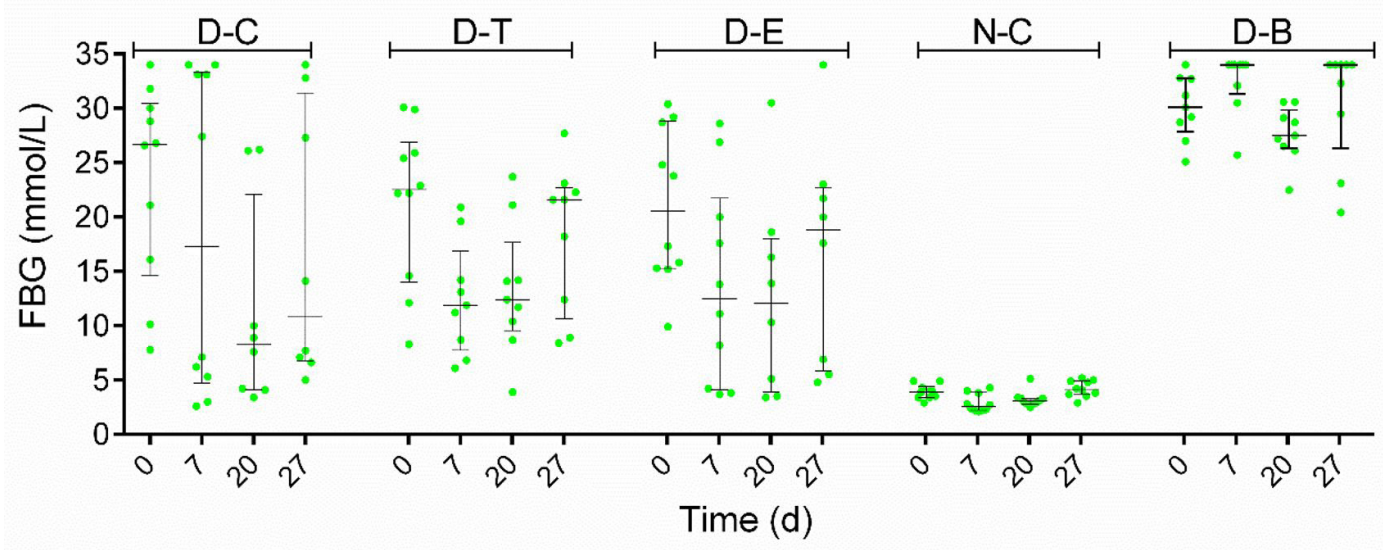

Figure 3. Effects of experimental diet supplemented with tea products on FBG of diabetic mice. Note: FBG, fasting blood glucose. The values were expressed as Median with interquartile range, the "-" symbol represents median value, and the upper and lower ranges of the box represent the $75 \%$ and $25 \%$ quartiles, respectively. Mice in diabetic group D-C fed a cornstarch-based diet, D-T fed a cornstarch-based functional diet supplemented with tea polyphenols, group D-E fed a cornstarch-based functional diet supplemented with EGCG, group D-B fed a basal diet, respectively ( $\mathrm{n}=8$ or 10$)$. Mice in normal group N-C fed a cornstarch-based functional diet $(\mathrm{n}=10)$. 
the experiment was $95.0 \%$. The purity of tea polyphenols used was $90.0 \%$, with $40.3 \%$ EGCG, $6.6 \%$ ECG, and $1.2 \%$ caffeine. Among them, EGCG is the most abundance and critical functional component. The current studies suggested that EGCG has so many health benefits. For example, EGCG act as an inhibitor, which can suppress the hydrolysis of starch by binding to active site of $\alpha$-amylase and $\alpha$-glucosidase (Zhang et al., 2018a; Li et al., 2018). EGCG can accelerate the utilization of glucose through attenuating insulin signalling blockade (Lin \& Lin, 2008; Bose et al., 2008), protect pancreatic islet cells (Song et al., 2003), and protect some other important organs, like kidney (Yoon et al., 2014; Cai et al., 2013; Periandavan et al., 2017) and liver (El-Mahalaway et al., 2013). EGCG also can reduce the absorptive of glucolipid in intestine tract (Nakai et al., 2005; Li et al., 2018), down-regulate the expression of sodiumdependent glucose cotransporter 1 (Hossain et al., 2002), promote glucose transporter 4 translocation in skeletal muscle (Ueda et al., 2008), attenuate insulin signaling blockade (Lin \& Lin, 2008) etc. Herein, this results suggested that tea's functional ingredients EGCG had a high utilizing value in improving glucose metabolism, and further reversing diabetes.

\subsection{Bacterial taxonomic differences analysis}

Analysis of flora changes at the bacterial phyla level

As Figure $4 \mathrm{a}$, b shows, analysis of the flora in fecal samples confifirmed changes involving 10 phyla, with Bacteroidetes,
Firmicutes, Proteobacteria and Actinobacteria being highly abundant and prevalent. These four phyla account for $98.38 \%$ of the reads in group D-C, $99.32 \%$ in D-E, $98.77 \%$ in D-T and $98.65 \%$ in $\mathrm{N}-\mathrm{C}$ of reads in groups $\mathrm{D}-\mathrm{C}, \mathrm{D}-\mathrm{E}, \mathrm{D}-\mathrm{T}$ and $\mathrm{N}-\mathrm{C}$, respectively.

As Figure 4c illustrates, Compared with group N-C, the levels of Proteobacteria, Candidatus Saccharibacteria and Cyanobacteria in group D-C were all significantly increased $(p<0.05)$, Tenericutes and Deferribacteres were all increased dramatically, but not reach the significant level $(p>0.05)$. Levels of Actinobacteria and Verrucomicrobia were significantly decreased by $78.75 \%$ $(p<0.05)$ and $35.24 \%(p<0.05)$, respectively.

More importantly, compared with group D-C, the levels of Candidatus Saccharibacteria and Cyanobacteria in group D-E were all decreased significantly $(p<0.05)$, but Actinobateria were increased significantly $(p<0.05)$. Moreover, levels of Cyanobacteria in group D-T were decreased by $40 \%(p>0.05)$, But levels of Actinobateria were significantly increased by $185.29 \%$ $(p<0.05)$. In addition, levels of Proteobacteria in group D-E and D-T were decreased by $30.63 \%$ and $40.87 \%$, respectively, but not significantly $(p>0.05)$.

\section{Analysis of flora changes at the bacterial family level}

As Figure 5a, b shows, twenty families demonstrated flora changes. The 10 most prominent were Porphyromonadaceae,
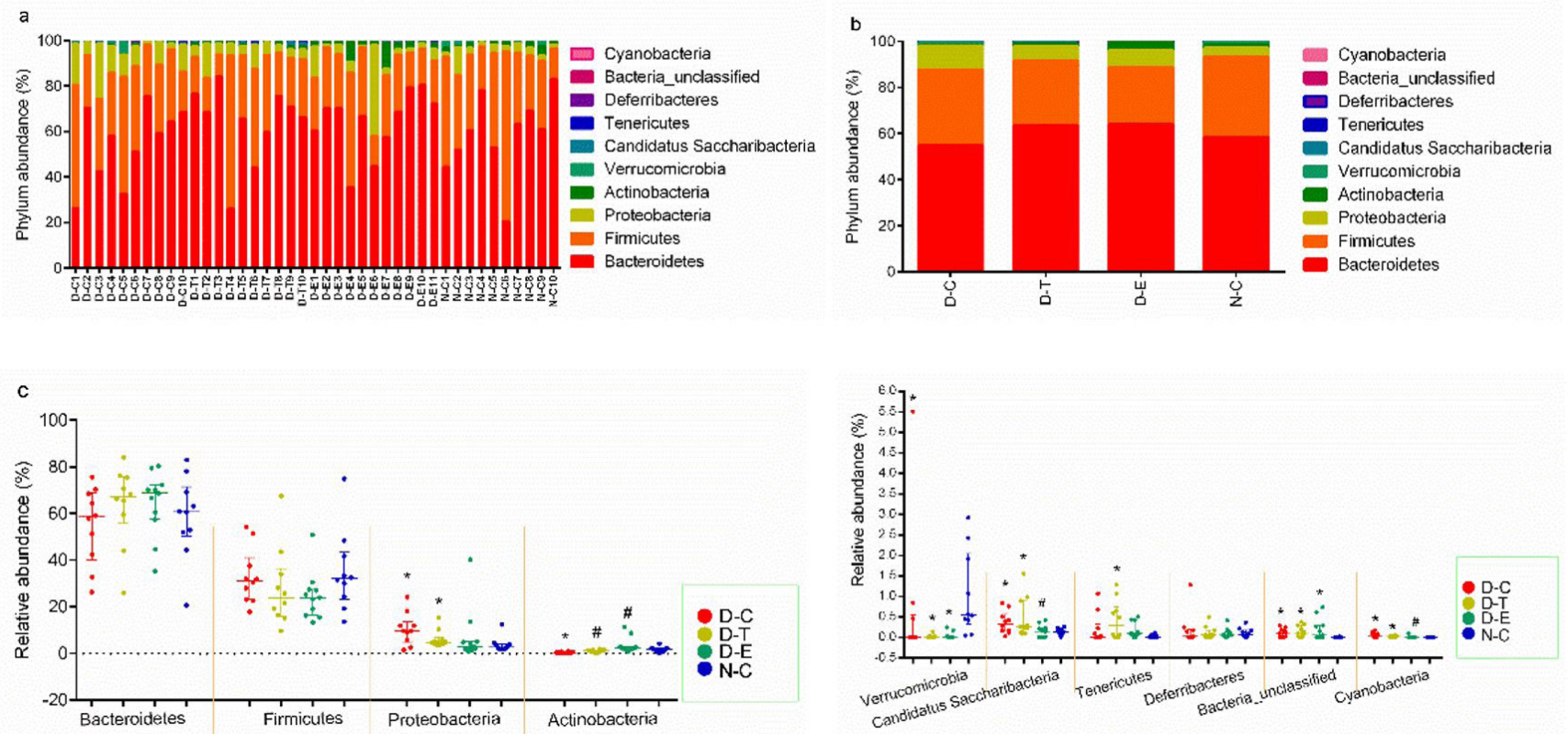

Figure 4. Effects of experimental diet supplemented with tea products on intestinal flora at the phyla levels in diabetic mice. Note: (a) Comparison of the abundances of bacterial phyla of each sample; (b) Comparison of the average abundance of each bacterial phyla in treatment groups, respectively; (c) Differences among the abundances of discriminatory phyla among four treatments. Values were express as Median with interquartile, each symbol represents a sample, and three horizontal line, respectively represent $3 / 4,1 / 2,1 / 4$ quantile from the top to bottom. $p$-values were calculated using the non-parametric Kruskal-Wallis test. " $*$ " represent significant difference $(p<0.05)$ were identified compared with group N-C, “\#” represent significant difference $(p<0.05)$ were identified compared with group D-C. Mice in diabetic group D-C fed a cornstarch-based diet, $\mathrm{D}$-T fed a cornstarch-based functional diet supplemented with tea polyphenols, group D-E fed a cornstarch-based functional diet supplemented with EGCG, respectively $(\mathrm{n}=8$ or 10$)$. Mice in normal group N-C fed a cornstarch-based functional diet $(\mathrm{n}=10)$. 

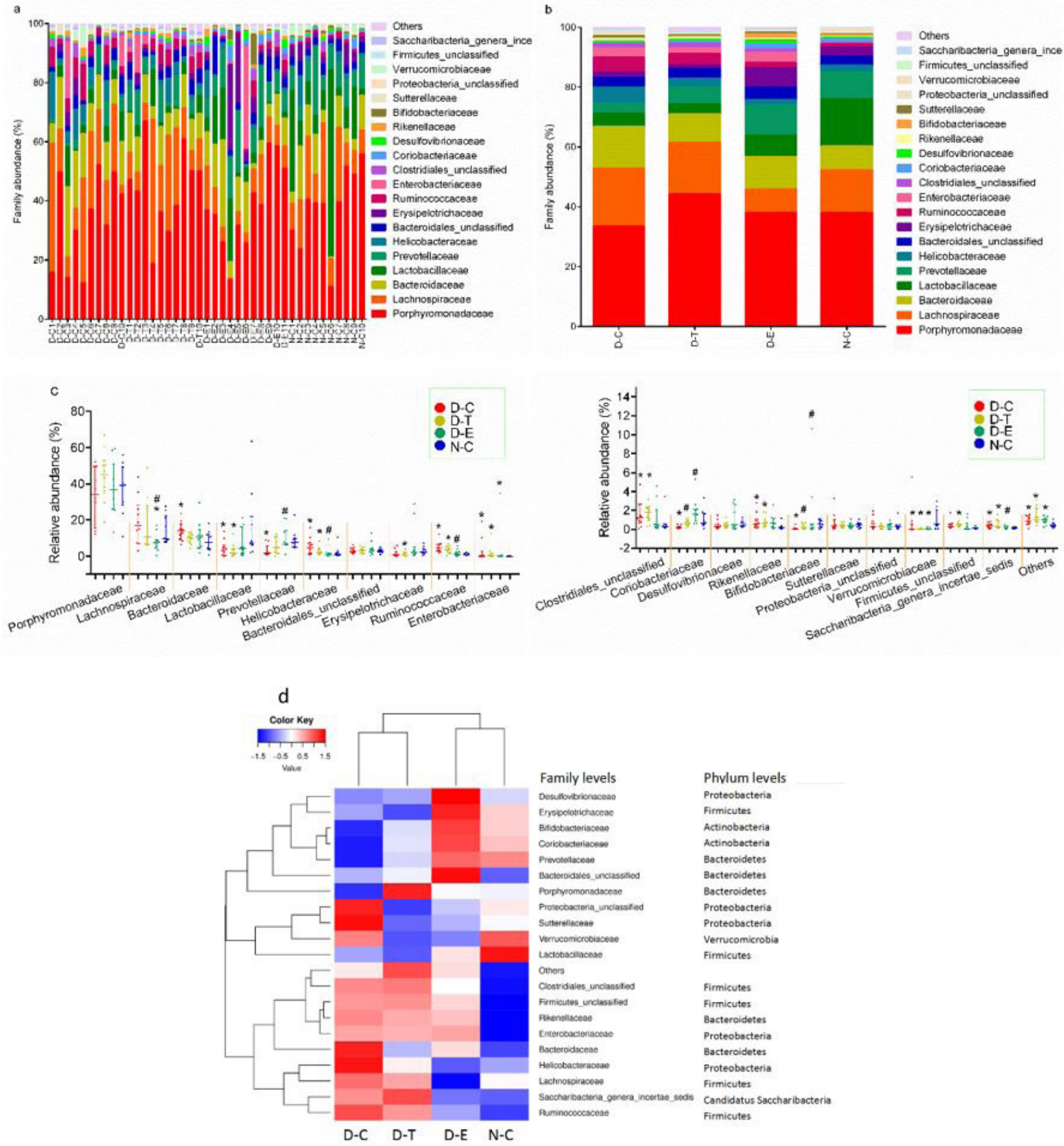

Figure 5. Effects of experimental diet supplemented with tea products on intestinal flora at the family levels in diabetic mice. Note: (a) Comparison of the abundances of bacterial family of each sample; (b) Comparison of the average abundance of each bacterial family in treatment groups, respectively; (c) Differences among the abundances of discriminatory family among four treatments. Values were express as Median with interquartile, each symbol represents a sample, and three horizontal line, respectively represent $3 / 4,1 / 2,1 / 4$ quantile from the top to bottom. $p$-values were calculated using the non-parametric Kruskal-Wallis test. “*” represent significant difference $(p<0.05)$ were identified compared with group N-C, “\#” represent significant difference $(p<0.05)$ were identified compared with group D-C. d. Heat maps of the top 20 microbial changes among treatments, For each group, the columns show the relative abundance data of the discriminatory flora at family levels listed to the right of the figure. The abundances of the family were clustered using unsupervised hierarchical clustering (blue, low abundance; red, high abundance). Mice in diabetic group D-C fed a cornstarch-based diet, D-T fed a cornstarch-based functional diet supplemented with tea polyphenols, group D-E fed a cornstarch-based functional diet supplemented with EGCG, respectively $(\mathrm{n}=8$ or 10). Mice in normal group N-C fed a cornstarch-based functional diet $(\mathrm{n}=10)$ 
Lachnospiraceae, Bacteroidaceae, Lactobacillaceae, Prevotellaceae, Helicobacteraceae, Bacteroidales_unclassified, Erysipelotrichaceae, Ruminococcaceae and Enterobacteriaceae (93.03\%, 91.41\%, $93.09 \%$ and $94.39 \%$ of reads in groups D-C, D-E, D-T and N-C, respectively).

Figure $5 \mathrm{c}$ shows that the levels of Bacteroidaceae, Helicobacteraceae, Ruminococcaceae, Enterobacteriaceae, Clostridiales_unclassified, Rikenellaceae and Saccharibacteria_genera_incertae_sedis in group D-C were all increased significantly $(p<0.05)$, Sutterellaceae, Proteobacteria_unclassified and Firmicutes_unclassified were increased by $53.19 \%, 52.63 \%$ and $327.17 \%$, but did not reach the significant level on statistics $(p>0.05)$. Levels of Prevotellaceae, Coriobacteriaceae, Bifidobacteriaceae and Verrucomicrobiaceae were all decreased significantly $(p<0.05)$, Lactobacillaceae and Erysipelotrichaceae were decreased by $71.79 \%$ and $45.88 \%$, but no significantly $(p>0.05)$ compared to health controls. When compared with group D-C, levels of Prevotellaceae and Coriobacteriaceae in group D-E were all increased significantly $(p<0.05)$, Lactobacillaceae, Erysipelotrichaceae and Desulfovibrionaceae were increased by $58.37 \%, 296.95 \%$ and $152.27 \%$, but all not significantly $(p>0.05)$. Levels of Lachnospiraceae, Helicobacteraceae, Ruminococcaceae and Saccharibacteria_genera_incertae_sedis were all decreased significantly $(p<0.05)$, Verrucomicrobiaceae were decreased by $92.65 \%$ ( $p>0.05)$. Moreover, levels of Coriobacteriaceae and Bifidobacteriaceae in group D-T were increased significantly $(p<0.05)$, Prevotellaceae were increased by $72.21 \%(p>0.05)$. Levels of Helicobacteraceae, Sutterellaceae, Proteobacteria_unclassified and Verrucomicrobiaceae were decreased by $47.44 \%, 48.61 \%, 58.62 \%$ and $95.59 \%$, but all not significantly $(p>0.05)$. Comparative analysis results show that diabetes disturbed the composition and micro-ecological balance of the intestinal flora, and that tea polyphenols and EGCG dietary supplements reversed the changes in levels at the bacterial phyla and family level.

Intestinal flora is closely related to body health, digestion and absorption, and intestinal flora dysbiosis cause metabolic building block, and eventual cause some disease (Lippert et al., 2017). In this study, diabetic mice showed intestinal flora dysbiosis because fecal flora reflect the ultimate result of interaction of entire intestinal flora and host. Diabetes showed increased levels of Rikenellaceae, Sutterellaceae and Helicobacteraceae. As we know, Rikenellaceae belongs to class Bacteroidia and phyla Bacteroidetes, which contains two genera, Rikenella and Alistipes. Sutterellaceae are Gram-negative, oxidase- and catalase-negative bacteria that can grow under microaerophilic or anaerobic conditions (Morotomi et al., 2011). In bacterial taxonomy, Sutterellaceae belongs to class Betaproteobacteria, and phyla Proteobacteria, and it contains families Sutterella and Parasutterella. Some members are indigenous intestinal flora in the gut of humans and other animals (Morotomi et al., 2011). In addition, significant changes of Helicobacteraceae has been linked to various diseases, including chronic gastritis (Oxley et al., 2004). Some species have been recognised as agents of gastrointestinal disease in both humans and a broad range of animal hosts by producing vacuolating cytotoxins, urease and cytotoxin-associated proteins that can damage bodily functions (Rasmi et al., 2009). Herein, an increase in family Helicobacteraceae may damage physiological functions or aggravate the morbidity of diabetes. Moreover, Firmicutes are the dominant bacterial community in the intestinal flora, accounting for $50-80 \%$ of all taxa (Jalanka-Tuovinen et al., 2011). In our intestines, Lachnospiraceae and Ruminococcaceae are the two prominent Firmicutes families. Studies suggest that Lachnospiraceae can protect colonic epithelial tissue (Duncan et al., 2002) by regulating the production of bacterial butyrate. Furthermore, some diseases such as Crohn's disease and inflammatory bowel disease are correlated with a markedly decreased levels of Lachnospiraceae (Fujimoto et al., 2013). But on the other hand, an increased levels of Lachnospiraceae and Ruminococcaceae may be not a good thing for diabetes. Lachnospiracea can decompose cellulose and hemicellulose components very well (Biddle et al., 2013), and Ruminococcaceae have unusual polysaccharide binding and degradation strategies (Flint et al., 2008). Ruminococcaceae is a short-chain fatty acid producer (Canfora et al., 2015). Thus, the increased absorption of small-molecule energy substances producted by Lachnospiraceae and Ruminococcaceae resulted in aggravation of blood glucose levels.

In addition, in diabetes there is significantly decreased relative abundance of Bifidobacteriaceae. Bifidobacteriaceae are anaerobic Gram-positive bacteria (phyla Actinobacteria). These species constituted a significant proportion of intestinal flora in humans and other animals. They can degrade glucose to acetic and lactic acid via characteristic fructose-6-phosphate shunt, in which fructose-6-phosphate phosphoketolase is the key enzyme (Mattarelli et al., 2014). They are well known for their special functions, such as maintaining a proper microbial balance in the gut, improving lactose tolerance, displaying anti-carcinogenic activity, and reducing serum cholesterol levels (Hennessy et al., 2016). Furthermore, members of Prevotellaceae can ferment carbohydrates and proteins very well, and produce acetate and $\mathrm{H}_{2}$ (Zhang et al., 2009). Prevotellaceae is one major families associated with secondary metabolite biosynthesis, transport, and catabolism, particularly participant in the transport of secondary metabolites. Thus, Prevotellaceae plays an important role in keeping balance of intestinal flora. Butyrate is a major energy source for colonic epithelial cells, and also it is closely related to gut microecological balance and intergrity of intestinal epithelium (Wu et al., 2018). The family Erysipelotrichaceae contains butyrate-producing bacteria. Therefore, the significantly decreased relative abundance of Prevotellaceae and Erysipelotrichaceae may cause some dysfunction in the intestines.

Tea polyphenols belongs to phenolic compounds (also called polyphenols). In this study, tea polyphenols and EGCG reversed the changes of intestinal flora compared to controls. Combining current researches, polyphenols have many active functions. It is very common and abundant in plants, like tea, fruits, vegetables etc. Once ingested, low-molecular polyphenols were partly absorbed in small intestinal, and the vast majority still remains in the intestinal tract, like largemolecular polyphenols (Appeldoorn et al., 2009). And the remaining were subjected to intestinal flora, and can been degraded into active small molecules (Kutschera et al., 2011; Filesi \& Benedetto, 2007). Current studies have found that polyphenols ingested can change intestinal flora, particularly changed the Bacteroides to Firmicutes ratio (Lee et al., 2006). Further studies found that special structure of catechin may affect microbial proliferation, particularly $(+)$ catechin significantly inhibited the growth of Clostridium, but 
promoted the growth of Bifidobacterium, Lactobacillus etc. (Tzounis et al., 2008). And some other studies found that tea polyphenols can inhibit the growth of pathogenic bacteria, like Clostridium perfringens, Bacteroides spp. etc., while have little affect on beneficial bacterium, such as Bifidobacterium, Lactobacillus. (Lee et al., 2006). Moreover, polyphenols extracted from different plants also have similar active functions, for example, flavan-3-ols (one kind of plant polyphenols) from grape seed can affect flora composition, such as increasing the Lactobacillus to Enterococcus ratio (Cueva et al., 2013). In this study, results suggest that tea polyphenols and EGCG play a role in reversing the changes of gut microbota induced by alloxan injection. By contrast, EGCG have a stronger regulatory effects on improving intestinal flora balance than that of tea polyphenols. Therefore, tea polyphenols and EGCG can really helps to promote nutritional value of functional food, improve intestinal health, and then helps to improve diabetes.

\section{Conclusion}

Diabetic mice had significant weight loss and serious hyperglycemia. CFD supplemented with tea polyphenols and EGCG resulted in a trend toward reduced FBG. More importantly, diabetes had significant intestinal flora disturbance, with particularly increased levels of Bacteroidaceae, Helicobacteraceae, Ruminococcaceae, Enterobacteriaceae, Rikenellaceae and Saccharibacteria_genera_incertae_sedis, and significantly decreased Prevotellaceae, Coriobacteriaceae, Bifidobacteriaceae and Verrucomicrobiaceae. CFD supplemented with EGCG significantly increased levels of Prevotellaceae and Coriobacteriaceae, decreased Lachnospiraceae, Helicobacteraceae, Ruminococcaceae and Saccharibacteria_genera_incertae_sedis. CFD supplemented with tea polyphenols significantly increased levels of Coriobacteriaceae and Bifidobacteriaceae. The comprehensive analysis indicated that tea polyphenols and EGCG supplementation improved the glucose metabolism, and reversed the sick changes of intestinal flora, suggesting tea polyphenols and EGCG are two valuable addition to promote nutritional value of CFD, which helps to improve diabetes patients.

\section{Conflict of interest}

No potential conflict of interest was reported by the authors.

\section{Funding}

This work was supported by the National Key Technology R\&D Program (2017YFD0400804) and Public Benefit Foundation of Zhejiang Province (LGN18C200010).

\section{Author contributions}

Jun Liu conceived and designed the experiments. YuLan Jiang, Yang-Jun Lv, Jun-Xian Pan, Yue-Jin Zhu, Jun Liu performed the experiments. Jun-Xian Pan, Jun Liu, Shi-Kang Zhang analyzed the data. Yue-Jin Zhu, Shi-Kang Zhang, Jun Liu contributed the reagents/materials/analysis tools. Jun Liu wrote the manuscript.

\section{Acknowledgements}

This work was supported by the National Key Technology R\&D Program (2017YFD0400804) and Public Benefit Foundation of Zhejiang Province (LGN18C200010).

\section{Reference}

Appeldoorn, M. M., Vincken, J. P., Gruppen, H., \& Hollman, P. C. H. (2009). Procyanidin dimers A1, A2, and B2 are absorbed without conjugation or methylation from the small intestine of rats. The Journal of Nutrition, 139(8), 1469-1473. http://dx.doi.org/10.3945/ jn.109.106765. PMid:19494022.

Baothman, O. A., Zamzami, M. A., Taher, I., Abubaker, J., \& Abu-Farha, M. (2016). The role of gut microbiota in the development of obesity and diabetes. Lipids in Health and Disease, 15(1), 108. http://dx.doi. org/10.1186/s12944-016-0278-4. PMid:27317359.

Biddle, A., Stewart, L., Blanchard, J., \& Leschine, S. (2013). Untangling the genetic basis of fibrolytic specialization by Lachnospiraceae and Ruminococcaceae in diverse gut communities. Diversity, 5(3), 627640. http://dx.doi.org/10.3390/d5030627.

Bose, M., Lambert, J. D., Ju, J., Reuhl, K. R., Shapses, S. A., \& Yang, C. S. (2008). The major green tea polyphenol, (-)-epigallocatechin-3gallate, inhibits obesity, metabolic syndrome, and fatty liver disease in high-fat-fed mice. The Journal of Nutrition, 138(9), 1677-1683. http://dx.doi.org/10.1093/jn/138.9.1677. PMid:18716169.

Cai, S., Zhong, Y., Li, Y., Huang, J., Zhang, J., Luo, G., \& Liu, Z. (2013). Blockade of the formation of insoluble ubiquitinated protein aggregates by EGCG3"Me in the alloxan-induced diabetic kidney. PLoS One, 8(9), e75687. http://dx.doi.org/10.1371/journal.pone.0075687. PMid:24098713.

Canfora, E. E., Jocken, J. W., \& Blaak, E. E. (2015). Short-chain fatty acids in control of body weight and insulin sensitivity. Nature Reviews. Endocrinology, 11(10), 577-591. http://dx.doi.org/10.1038/ nrendo.2015.128. PMid:26260141.

Caporaso, J. G., Bittinger, K., Bushman, F. D., Desantis, T. Z., Andersen, G. L., \& Knight, R. (2010). PyNAST: a flexible tool for aligning sequences to a template alignment. Bioinformatics, 26(2), 266-267. http://dx.doi.org/10.1093/bioinformatics/btp636. PMid:19914921.

Collins, Q. F., Liu, H. Y., Pi, J., Liu, Z., Quon, M. J., \& Cao, W. (2007). Epigallocatechin-3-gallate (EGCG), a green tea polyphenol, suppresses hepatic gluconeogenesis through $5^{\prime}$-amp-activated protein kinase. The Journal of Biological Chemistry, 282(41), 30143-30149. http:// dx.doi.org/10.1074/jbc.M702390200. PMid:17724029.

Cueva, C., Sánchez-Patán, F., Monagas, M., Walton, G. E., Gibson, G. R., Martín-álvarez, P. J., Bartolomé, B., \& Moreno-Arribas, M. V. (2013). In vitro, fermentation of grape seed flavan-3-ol fractions by human faecal microbiota: changes in microbial groups and phenolic metabolites. FEMS Microbiology Ecology, 83(3), 792-805. http:// dx.doi.org/10.1111/1574-6941.12037. PMid:23121387.

Damasceno, D. C., Volpato, G. T., Calderon, I. M. P., \& Cunha Rudge, M. V. (2002). Oxidative stress and diabetes in pregnant rats. Animal Reproduction Science, 72(3-4), 235-244. http://dx.doi.org/10.1016/ S0378-4320(02)00094-5. PMid:12137985.

Duncan, S. H., Barcenilla, A., Stewart, C. S., Pryde, S. E., \& Flint, H. J. (2002). Acetate utilization and butyryl coenzyme A (CoA): acetateCoA transferase in butyrate-producing bacteria from the human large intestine. Applied and Environmental Microbiology, 68(10), 5186-5190. http://dx.doi.org/10.1128/AEM.68.10.5186-5190.2002. PMid:12324374. 
El-Mahalaway, A. M., El-Gohary, O. A., \& Wahba, O. (2013). The histopathological and biochemical effects of green tea extract on the liver of high-fat diet and streptozotocin diabetic female rats. Journal of Evidence-Based Women's Health Journal Society, 3(1), 25-34. http://dx.doi.org/10.1097/01.EBX.0000419543.86961.00.

Filesi, C., \& Benedetto, R. D. (2007). Polyphenols, dietary sources and bioavailability. Annali Dellistituto Superiore Di Sanità, 43(4), 348-361.

Flint, H. J., Bayer, E. A., Rincon, M. T., Lamed, R., \& White, B. A. (2008). Polysaccharide utilization by gut bacteria: potential for new insights from genomic analysis. Nature Reviews. Microbiology, 6(2), 121-131. http://dx.doi.org/10.1038/nrmicro1817. PMid:18180751.

Fujimoto, T., Imaeda, H., Takahashi, K., Kasumi, E., Bamba, S., Fujiyama, Y., \& Andoh, A. (2013). Decreased abundance of Faecalibacterium prausnitzii in the gut microbiota of crohn's disease. Journal of Gastroenterology and Hepatology, 28(4), 613-619. http://dx.doi. org/10.1111/jgh.12073. PMid:23216550.

Gomes, A. C., Hoffmann, C., \& Mota, J. F. (2018). The human gut microbiota: metabolism and perspective in obesity. Gut Microbes, 9(4), 308-325. http://dx.doi.org/10.1080/19490976.2018.1465157 . PMid:29667480.

Hennessy, A. A., Ross, P., Devery, R., \& Stanton, C. (2016). Bifidobacterially produced, $c 18: 3$ and $c 18: 4$ conjugated fatty acids exhibit in vitro anti-carcinogenic and anti-microbial activity. European Journal of Lipid Science and Technology, 118(11), 1743-1758. http://dx.doi. org/10.1002/ejlt.201500424.

Hossain, S. J., Kato, H., Aoshima, H., Yokoyama, T., Yamada, M., \& Hara, Y. (2002). Polyphenol-induced inhibition of the response of $\mathrm{na}(+) /$ glucose cotransporter expressed in xenopus oocytes. Journal of Agricultural and Food Chemistry, 50(18), 5215-5219. http://dx.doi. org/10.1021/jf020252e. PMid:12188632.

Huang, Q., Jiang, H., Briggs, B. R., Wang, S., Hou, W., Li, G., Wu, G., Solis, R., Arcilla, C. A., Abrajano, T., \& Dong, H. (2013). Archaeal and bacterial diversity in acidic to circumneutral hot springs in the philippines. FEMS Microbiology Ecology, 85(3), 452-464. http:// dx.doi.org/10.1111/1574-6941.12134. PMid:23607726.

Jalanka-Tuovinen, J., Salonen, A., Nikkilä, J., Immonen, O., Kekkonen, R., Lahti, L., Palva, A., \& Vos, W. M. (2011). Intestinal microbiota in healthy adults, temporal analysis reveals individual and common core and relation to intestinal symptoms. PLoS One, 6(7), e23035. http://dx.doi.org/10.1371/journal.pone.0023035. PMid:21829582.

Kutschera, M., Engst, W., Blaut, M., \& Braune, A. (2011). Isolation of catechin-converting human intestinal bacteria. Journal of Applied Microbiology, 111(1), 165-175. http://dx.doi.org/10.1111/j.13652672.2011.05025.x. PMid:21457417.

Lee, H. C., Jenner, A. M., Low, C. S., \& Lee, Y. K. (2006). Effect of tea phenolics and their aromatic fecal bacterial metabolites on intestinal microbiota. Research in Microbiology, 157(9), 876-884. http://dx.doi. org/10.1016/j.resmic.2006.07.004. PMid:16962743.

Li, X., Li, S. Y., Chen, M., Wang, J. Y., Xie, B. J., \& Sun, Z. D. (2018). (-)-epigallocatechin-3-gallate (EGCG) inhibits starch digestion and improves glucose homeostasis through direct or indirect activation of pxr/car-mediated phaseIImetabolism in diabetic mice. Food \& Function, 9(9), 4651-4663. http://dx.doi.org/10.1039/C8FO01293H. PMid:30183039.

Lin, C. L., \& Lin, J. K. (2008). Epigallocatechin gallate (EGCG) attenuates high glucose-induced insulin signaling blockade in human Hepg2 hepatoma cells. Molecular Nutrition \& Food Research, 52(8), 930939. http://dx.doi.org/10.1002/mnfr.200700437. PMid:18496818.

Lippert, K., Kedenko, L., Antonielli, L., Kedenko, I., Gemeier, C., Leitner, M., Kautzky-Willer, A., Paulweber, B., \& Hackl, E. (2017). Gut microbiota dysbiosis associated with glucose metabolism disorders and the metabolic syndrome in older adults. Beneficial Microbes, 8(4), 545-556. http://dx.doi.org/10.3920/BM2016.0184. PMid:28701081.

Mattarelli, P., Brandi, G., Calabrese, C., Fornari, F., Prati, G. M., Biavati, B., \& Sgorbati, B. (2014). Occurrence of bifidobacteriaceae in human hypochlorhydria stomach. Microbial Ecology in Health and Disease, 25(1), 1-7.PMid:24427111.

Morotomi, M., Nagai, F., \& Watanabe, Y. (2011). Parasutterella secunda $s p$. nov. isolated from human faeces and proposal of Sutterellaceae fam. nov. in the order burkholderiales. International Journal of Systematic and Evolutionary Microbiology, 61(3), 637-643. http:// dx.doi.org/10.1099/ijs.0.023556-0. PMid:20400667.

Nakai, M., Fukui, Y., Asami, S., Toyoda-Ono, Y., Iwashita, T., Shibata, H., Mitsunaga, T., Hashimoto, F., \& Kiso, Y. (2005). Inhibitory effects of oolong tea polyphenols on pancreatic lipase in vitro. Journal of Agricultural and Food Chemistry, 53(11), 4593-4598. http://dx.doi. org/10.1021/jf047814+. PMid:15913331.

Namkoong, C., Kim, M. S., Jang, P. G., Han, S. M., Park, H. S., Koh, E. H., Lee, W. J., Kim, J. Y., Park, I. S., Park, J. Y., \& Lee, K. U. (2005). Enhanced hypothalamic amp-activated protein kinase activity contributes to hyperphagia in diabetic rats. Diabetes, 54(1), 63-68. http://dx.doi.org/10.2337/diabetes.54.1.63. PMid:15616011.

Nugent, A. P. (2005). Health properties of resistant starch. Nutrition Bulletin, 30(1), 27-54. http://dx.doi.org/10.1111/j.1467-3010.2005.00481.x.

Oxley, A. P. A., Powell, M., \& Mckay, D. B. (2004). Species of the family Helicobacteraceae detected in an australian sea lion (neophoca cinerea) with chronic gastritis. Journal of Clinical Microbiology, 42(8), 3505-3512. http://dx.doi.org/10.1128/JCM.42.8.3505-3512.2004. PMid:15297490.

Qiu, S., Yadav, M. P., Zhu, Q., Chen, H., Liu, Y., \& Yin, L. (2017). The addition of corn fiber gum improves the long-term stability and retrogradation properties of corn starch. Journal of Cereal Science, 76, 92-98. http://dx.doi.org/10.1016/j.jcs.2017.04.012.

Periandavan, K., Mohan, T., Velusamy, P., Chakrapani, L. N., \& Johnson, T. (2017). Impact of EGCG supplementation on the progression of diabetic nephropathy in rats: an insight into fibrosis and apoptosis. Journal of Agricultural and Food Chemistry, 65(36), 8028-8036.

Rasmi, Y., Sadreddini, M., Jamali, M., Peirouvi, T., \& Khosravifar, F. (2009). Frequency of cytotoxin associated gene a $(+)$ Helicobacter pylori in peptic ulcer disease: difference between gastric and duodenal ulcer disease. Journal of Medical Sciences, 9(3), 146-150. http://dx.doi.org/10.3923/jms.2009.146.150.

Rognes, T., Flouri, T., Nichols, B., Quince, C., \& Mahé, F. (2016). Vsearch: a versatile open source tool for metagenomics. PeerJ, 4(10), e2584. http://dx.doi.org/10.7717/peerj.2584. PMid:27781170.

Sae-Tan, S., Grove, K. A., Kennett, M. J., \& Lambert, J. D. (2011). (-)-epigallocatechin-3-gallate increases the expression of genes related to fat oxidation in the skeletal muscle of high fat-fed mice. Food \& Function, 2(2), 111-116. http://dx.doi.org/10.1039/c0fo00155d. PMid:21779555.

Song, E. K., Hur, H., \& Han, M. K. (2003). Epigallocatechin gallate prevents autoimmune diabetes induced by multiple low doses of streptozotocin in mice. Archives of Pharmacal Research, 26(7), 559-563. http://dx.doi.org/10.1007/BF02976881. PMid:12934649.

Stefano, G. B., Challenger, S., \& Kream, R. M. (2016). Hyperglycemiaassociated alterations in cellular signaling and dysregulated mitochondrial bioenergetics in human metabolic disorders. European Journal of Nutrition, 55(8), 2339-2345. http://dx.doi.org/10.1007/ s00394-016-1212-2. PMid:27084094.

Tzounis, X., Vulevic, J., Kuhnle, G. G. C., George, T., Leonczak, J., Gibson, G. R., Kwik-Uribe, C., \& Spencer, J. P. E. (2008). Flavanol 
monomer induced changes to the human fecal microflora. The British Journal of Nutrition, 99(4), 782-792. http://dx.doi.org/10.1017/ S0007114507853384. PMid:17977475.

Ueda, M., Nishiumi, S., Nagayasu, H., Fukuda, I., Yoshida, K., \& Ashida, H. (2008). Epigallocatechin gallate promotes glut 4 translocation in skeletal muscle. Biochemical and Biophysical Research Communications, 377(1), 286-290. http://dx.doi.org/10.1016/j.bbrc.2008.09.128. PMid:18845128.

Wu, X., Wu, Y., He, L., Wu, L., Wang, X., \& Liu, Z. (2018). Effects of the intestinal microbial metabolite butyrate on the development of colorectal cancer. Journal of Cancer, 9(14), 2510-2517. http://dx.doi. org/10.7150/jca.25324. PMid:30026849.

Yoon, S. P., Maeng, Y. H., Hong, R., Lee, B. R., Kim, C. G., Kim, H. L., Chung, J. H., \& Shin, B. C. (2014). Protective effects of epigallocatechin gallate (egcg) on streptozotocin-induced diabetic nephropathy in mice. Acta Histochemica, 116(8), 1210-1215. http://dx.doi.org/10.1016/j. acthis.2014.07.003. PMid:25154791.

Zhang, H. H., Liu, J., Lv, Y. J., Jiang, Y. L., Pan, J. X., Zhu, Y. J., Huang, M. G., \& Zhang, S. K. (2020). Changes in intestinal microbiota of type 2 diabetes in mice in response to dietary supplementation with instant tea or matcha. Canadian Journal of Diabetes, 44(1), 44-52. http://dx.doi.org/10.1016/j.jcjd.2019.04.021. PMid:31378691.

Zhang, H., Dibaise, J. K., Zuccolo, A., Kudrna, D., Braidotti, M., Yu, Y., Parameswaran, P., Crowell, M. D., Wing, R., Rittmann, B. E., \& Krajmalnik-Brown, R. (2009). Human gut microbiota in obesity and after gastric bypass. Proceedings of the National Academy of Sciences of the United States of America, 106(7), 2365-2370. http:// dx.doi.org/10.1073/pnas.0812600106. PMid:19164560.

Zhang, H., Jiang, Y., Pan, J., Lv, Y., Liu, J., Zhang, S., \& Zhu, Y. (2018a). Effect of tea products on the in vitro enzymatic digestibility of starch. Food Chemistry, 243, 345-350. http://dx.doi.org/10.1016/j. foodchem.2017.09.138. PMid:29146347.

Zhang, Y., Tang, K., Deng, Y., Chen, R., Liang, S., Xie, H., He, Y., Chen, Y., \& Yang, Q. (2018b). Effects of shenling baizhu powder herbal formula on intestinal microbiota in high-fat diet-induced nafld rats. Biomedicine and Pharmacotherapy, 102, 1025-1036. http://dx.doi. org/10.1016/j.biopha.2018.03.158. PMid:29710519.. 